\title{
Structured reporting in neuro-oncological imaging: achieving reliable prediction of molecular subtypes in glioma based on pre-treatment multi-sequence MRI
}

\author{
Nico Sollmann ${ }^{1,2,3}$ (D) \\ Received: 31 March 2021 / Accepted: 8 April 2021 / Published online: 7 August 2021 \\ (C) The Author(s) 2021
}

Structured reporting commonly refers to the use of predefined formats and terms to create radiological reports, using a high level of standardized and organized information in template context $[1,2]$. In a recent publication, the European Society of Radiology (ESR) proposed the triad of quality, datafication/ quantification, and accessibility as a major requirement for the transition from conventional "free-text" reporting to structured reporting in radiology [3]. First, improvement of quality for radiological reporting may be achieved through standardization, which can be established by implementation of templates that provide a sort of checklist of items that need to be included in the report, commonly in a predefined order. Second, datafication/quantification refers to the inclusion of specific data elements (e.g., grading systems like the TNM classification) and quantitative parameters (e.g., radiomics or image-based biomarkers) in the report. Third, availability plays an essential role for broad consideration of radiological information with multifarious purposes including diagnosis, clinical decision-making, and transfer in order to target research questions or generate representative databases for specific pathologies or imaging findings.

The study by Nam et al [4], published in European Radiology, aims at identifying reproducible parameters de-

This comment refers to the article available at https://doi.org/10.1007/ s00330-021-08015-4.

Nico Sollmann

Nico.Sollmann@tum.de

1 Department of Diagnostic and Interventional Radiology, University Hospital Ulm, Albert-Einstein-Allee 23, 89081 Ulm, Germany

2 Department of Diagnostic and Interventional Neuroradiology, School of Medicine, Klinikum rechts der Isar, Technical University of Munich, Ismaninger Str. 22, 81675 Munich, Germany

3 TUM-Neuroimaging Center, Klinikum rechts der Isar, Technical University of Munich, Munich, Germany rived from cranial magnetic resonance imaging (MRI) that are predictive of molecular subtypes in glioma using a structured reporting system. Specifically, in this structured reporting system, MRI-derived parameters including tumor necrosis and margin, edema, internal cyst, T2/fluidattenuated inversion recovery (FLAIR) mismatch, contrast enhancement patterns, and cerebral blood volume (CBV) higher than normal cortex were considered, which have been derived from multi-sequence scanning with non-contrast and contrastenhanced T1- and T2-weighted, and FLAIR sequences as well as CBV maps. These image datasets were visually analyzed, and structured reports were created by three neuroradiologists with different experience levels ( 1 to 7 years) in a training set of 141 and a validation set of 131 patients with glioma. Included patients were stratified into five risk types considering pathomolecular evaluation with isocitrate dehydrogenase (IDH) mutation and 1p19q co-deletion status of glioma, similar to the risk types proposed previously [5]. The main finding is that in the validation set, the prediction of three of the five defined risk types showed high diagnostic performance regarding predominant enhancement (for IDH-wildtype glioblastoma), T2/FLAIR mismatch sign and no necrosis (for IDH-mutant diffuse astrocytoma, grades II/III), and internal cyst or necrosis (for IDH-mutant oligodendroglioma, 1p19q co-deleted). Thus, by means of structured reporting, the study was successful in identifying imaging parameters in pretreatment MRI that would facilitate classification of some glioma entities. Of note, diagnostic modeling to achieve these results considered the MRI-based parameters that achieved an agreement of $>50 \%$ among the three readers, and final diagnosis was based on pathomolecular evaluation using tumor tissue probes.

This study represents an appealing way to implement structured reporting to address a common diagnostic issue in neuroradiology. Extracting parameters from pretreatment MRI acquisitions has the potential to facilitate 
early non-invasive diagnosis and tailored treatment decision-making. Specifically, it has been suggested previously that treatment approaches may be associated with certain characteristics observed in pre-treatment MRI of glioma, with the choice of neurosurgical intervention being significantly related to the complexity of tumor infiltration and contrast enhancement patterns since patients whose lesions had indiscernible contrast enhancement received a more conservative management by approximately three times longer intervals between MRI acquisitions and surgery [6]. In this regard, follow-up studies may extend the present work of Nam et al [4] by also including further sequences that are increasingly applied in clinical imaging protocols, such as diffusion tensor imaging (DTI) or alternative T1-weighted sequences to standardly used turbo field-echo imaging. Specifically, DTI may further facilitate discrimination between tumor entities according to IDH status since DTI-derived fractional anisotropy (FA) values were significantly different between oligodendroglial tumors with IDH mutations and those without mutations, for instance [7, 8]. Furthermore, implementation of 3D black-blood turbo spin-echo sequences could facilitate clear depiction of tumor-related contrast enhancement, tumor borders, and satellite lesions that are not or only inferiorly visualized in conventional T1weighted imaging $[9,10]$.

In agreement with the recommendations of the ESR, the present study by Nam et al [4] comprehensively used structured reporting that fulfills the criteria of quality through standardization and accessibility, with the structured reports being the basis of predictive modeling targeting glioma stratification. With respect to datafication/quantification, clear categories for structured reporting within a template have been defined by the authors. Beyond the scope of this study is the additional use of quantitative MRI-based data, which is commonly not extracted and not routinely available in the current clinical setup for neuro-oncological imaging. The next step based on this study's promising results and also similar studies for other body regions would be to accomplish seamless availability of quantitative parameters for structured reporting in the routine setup. Here, both increasingly used sequences that allow extraction of meaningful values (e.g., DTI with extraction of FA or other scalar measures) as well as radiomics could ideally refine pre-treatment MRI-based prediction of tumor entities in the future, supplementing standardized visual image reading by objective metrics from quantitative MRI. However, besides achieving a workable solution that effectively combines visual image reading with quantitative parameters, integration into the radiologic workflow and existing technical infrastructure is key for practicability in daily clinical routine. Once further steps of integration have been taken, structured reporting fusing visual image reading with quantitative parameters certainly has high potential to considerably advance radiological reporting, and to get far more information out of contemporary MRI acquisitions.

In summary, the work by Nam et al [4] comes timely and can be interpreted as a clear advocate for speeding up integration of structured reporting into the clinical workflow, given this study's comprehensible results based on pre-treatment MRI that may, at least for some glioma entities, augur correct tumor classification with reasonable diagnostic accuracy. The consideration of independent training and validation datasets as well as imaging parameters that adhere to a certain threshold of inter-reader agreement speak for the robustness and quality of this study.

Funding Open Access funding enabled and organized by Projekt DEAL.

\section{Compliance with ethical standards}

Guarantor The scientific guarantor of this publication is Nico Sollmann, MD, PhD.

Conflict of interest The author of this manuscript declares no relationships with any companies, whose products or services may be related to the subject matter of the article.

Statistics and biometry No statistical methods were necessary for this manuscript.

Informed consent Not applicable.

Ethical approval Not applicable.

Methodology Editorial comment.

Open Access This article is licensed under a Creative Commons Attribution 4.0 International License, which permits use, sharing, adaptation, distribution and reproduction in any medium or format, as long as you give appropriate credit to the original author(s) and the source, provide a link to the Creative Commons licence, and indicate if changes were made. The images or other third party material in this article are included in the article's Creative Commons licence, unless indicated otherwise in a credit line to the material. If material is not included in the article's Creative Commons licence and your intended use is not permitted by statutory regulation or exceeds the permitted use, you will need to obtain permission directly from the copyright holder. To view a copy of this licence, visit http://creativecommons.org/licenses/by/4.0/.

\section{References}

1. Liu D, Zucherman M, Tulloss WB Jr (2006) Six characteristics of effective structured reporting and the inevitable integration with speech recognition. J Digit Imaging 19:98-104 
2. Kahn CE Jr, Langlotz CP, Burnside ES et al (2009) Toward best practices in radiology reporting. Radiology 252:852-856

3. European Society of Radiology (2018) ESR paper on structured reporting in radiology. Insights Imaging 9:1-7

4. Nam YK, Park JE, Park SY et al (2021) Reproducible imagingbased prediction of molecular subtype and risk stratification of gliomas across different experience levels using a structured reporting system. Eur Radiol. https://doi.org/10.1007/s00330-021-08015-4

5. Eckel-Passow JE, Lachance DH, Molinaro AM et al (2015) Glioma groups based on $1 \mathrm{p} / 19 \mathrm{q}, \mathrm{IDH}$, and TERT promoter mutations in tumors. N Engl J Med 372:2499-2508

6. Due-Tonnessen P, Pinho MC, Emblem KE et al (2019) The impact of MRI features and observer confidence on the treatment decisionmaking for patients with untreated glioma. Sci Rep 9:19898

7. Xiong J, Tan W, Wen J et al (2016) Combination of diffusion tensor imaging and conventional MRI correlates with isocitrate dehydrogenase $1 / 2$ mutations but not $1 \mathrm{p} / 19 \mathrm{q}$ genotyping in oligodendroglial tumours. Eur Radiol 26:1705-1715
8. Xiong J, Tan WL, Pan JW et al (2016) Detecting isocitrate dehydrogenase gene mutations in oligodendroglial tumors using diffusion tensor imaging metrics and their correlations with proliferation and microvascular density. J Magn Reson Imaging 43:45-54

9. Finck T, Gempt J, Zimmer C, Kirschke JS, Sollmann N (2021) MR imaging by $3 \mathrm{D}$ T1-weighted black blood sequences may improve delineation of therapy-naive high-grade gliomas. Eur Radiol 31: 2312-2320

10. Kammer NN, Coppenrath E, Treitl KM, Kooijman H, Dietrich O, Saam T (2016) Comparison of contrast-enhanced modified T1weighted 3D TSE black-blood and 3D MP-RAGE sequences for the detection of cerebral metastases and brain tumours. Eur Radiol 26:1818-1825

Publisher's note Springer Nature remains neutral with regard to jurisdictional claims in published maps and institutional affiliations. 